\title{
La reivindicación de la política económica y la equidad en un mundo globalizado*
}

\author{
Vindicating economic policy and equity \\ in a global world
}

\author{
Ricardo Ffrench-Davis ${ }^{\star \star}$
}

\section{Resumen}

América Latina no solo está lejos de alcanzar el desarrollo sino que se caracteriza por una enorme brecha en materia de equidad. En el contexto de la fuerte tendencia a la globalización, el artículo plantea la necesidad de alcanzar el crecimiento con equidad mediante políticas públicas que establezcan condiciones favorables para que la ella tenga un rostro social. Reseña brevemente los alcances económicos de la globalización y centra la atención en los compromisos asumidos por la comunidad internacional para mejorar la gobernabilidad mundial y lograr que el desarrollo beneficie a todos por igual. Dentro de ellos destaca la Iniciativa para la Acción contra el Hambre y la Pobreza cuya finalidad es

Texto basado en la exposición realizada en el Seminario de la Fundación F. Ebert sobre «Globalización con rostro social -la revaloración de lo nacional a través de los impuestos globales», y en planteamientos expuestos en dos reuniones del Consejo Económico y Social realizadas en Nueva York y en Brasilia.

** Profesor de Economía de la Universidad de Chile.

Las opiniones expresadas son de exclusiva responsabilidad del autor, quien agradece los valiosos comentarios de Luis Eduardo Escobar, de otros miembros del Grupo Chile del GT-7, y los recibidos en el Seminario de la FES. 
identificar fuentes innovadoras de financiamiento de proyectos que faciliten la consecución de los Objetivos de Desarrollo del Milenio.

Palabras clave: globalización, crecimiento, equidad, GT-7, UNITAID

Abstract

The Latin America region is not only far from having reached development; it also has an enormous equity gap. In the context of the growing trend towards globalization, the article deals with the need to combine growth with equity through public policies that will make it possible to establish the required conditions for endowing growth with a social dimension. It touches briefly upon globalization's economic implications and focuses on commitments assumed by the international community in order to improve global governance and allow development to be equally favourable to all. Among these, reference is made to the Action Initiative against Hunger and Poverty, which aims at identifying innovative financing sources that will facilitate the achievement of Millennium Development Goals.

Keywords: globalization, growth, equity, GT-7, UNITAID. 


\section{INTRODUCCIÓN}

América Latina, con toda su heterogeneidad, es una región que está aún muy lejos del desarrollo económico, y en la que hay una notable desigualdad. $\mathrm{Su}$ ingreso por habitante (PIB p/c) es apenas un cuarto de aquel de las economías más desarrolladas. Refuerza la gravedad de esa distancia la enorme bre- cha de equidad al interior de cada uno de nuestros países ${ }^{1}$.

En efecto en América Latina ella más que duplica la de los países desarrollados que constituyen el G-7 (gráfico 1). En consecuencia, se precisa aumentar la torta, pero también mejorar su distribución. Esto es, crecer con equidad.

\section{GRÁFICO I}

AmÉrica Latina y países desarRollados, PIB P/C y Distribución DEL INGRESO, 2005 (DÓLARES PPP)

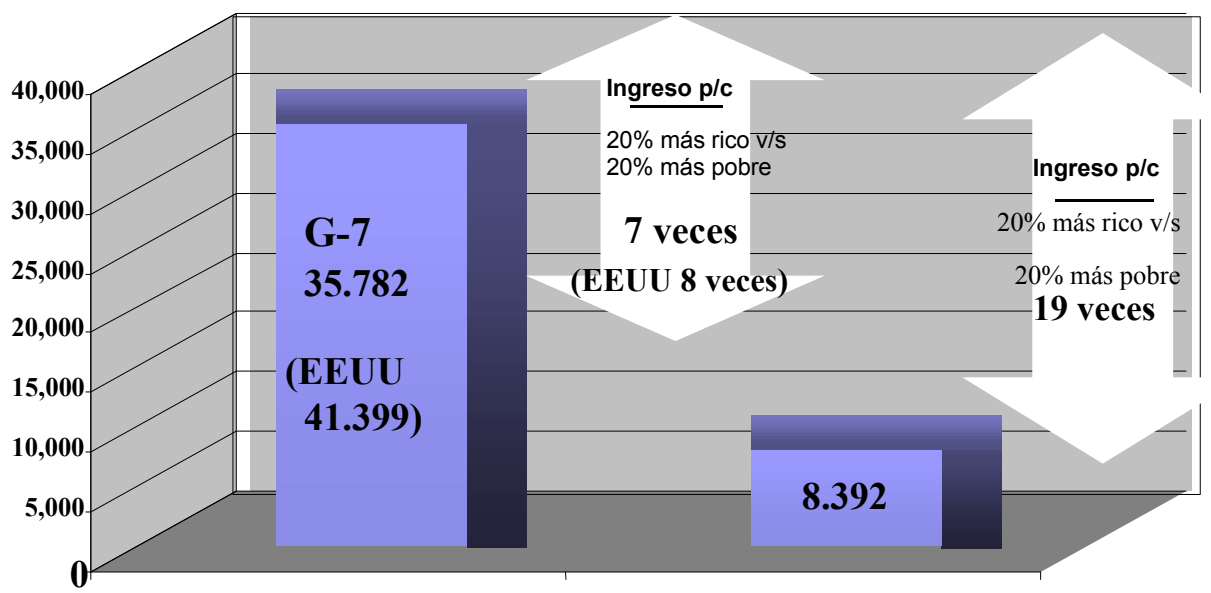

Países Desarrollados (G-7) América Latina (19)

Fuentes: Para PIB per cápita, Fondo Monetario Internacional, World Economic Outlook Database (2006). Para distribución del ingreso, Banco Mundial, World Development Indicators Database (2006). El grupo de los siete (G-7) incluye Alemania, Canadá, Estados Unidos, Francia, Italia, Japón y Reino Unido.

¿Cómo se logran los dos objetivos de crecimiento con equidad? El planteamiento solo es viable si hay éxito sostenido en ambas dimensiones, si las políticas públicas logran que sean complementarias. Al crecer con equidad, se distribuyen los ingresos y las utilidades entre más empresarios pequeños y me-

1 Excepciones, como países latinoamericanos más equitativos, son Costa Rica y Uruguay. 
dianos, y trabajadores de diversas calificaciones, con remuneraciones crecientes a través del tiempo.

Estos objetivos están inmersos en un contexto en el que nuestros países enfrentan una fuerte tendencia a la globalización. Ella, naturalmente, restringe el espacio para las políticas nacionales. Lo restringe, pero no lo elimina si hay voluntad de HACER globalización, de ser actor y no sujeto pasivo. Voluntad de intervenir activamente hacia el interior, con políticas públicas activas y coherentes; $y$ hacia el exterior, como países pequeños y/o en desarrollo, de actuar unidos en su región y con otras naciones para mejorar la gobernabilidad global y para corregir las normas internacionales, en procura de «nivelar» el terreno para el comercio, las finanzas y la innovación en las economías en desarrollo.

En esta exposición, hago un breve recuento de los alcances económicos de la globalización, y luego me concentro en presentar una iniciativa internacional de un grupo de países del sur y del norte para avanzar hacia una globalización con rostro social.

\section{GLOBALIZACIÓN ECONÓMICA Y ESPACIO PARA LO LOCAL}

La globalización no es un paquete único: es un conjunto complejo de componentes con distintos beneficios y costos y cuya dosificación varía con el nivel de desarrollo y con las políticas públicas de cada país. Hay que distin- guir entre esos componentes, sus costos y beneficios, porque así será posible navegar eficientemente en la globalización, cosechando más beneficios y menos costos. La proporción entre ambos no está predeterminada. Depende de cómo cada país trate de maximizar beneficios y minimizar costos, de cuánto entienda del proceso y de cuáles sean los intereses que predominen.

En el ámbito más restringido de lo económico, la globalización no es un proceso homogéneo. Hay diversas dimensiones, que se han movido a distintas velocidades en los últimos años. A su vez, desde la perspectiva de los países en desarrollo, la globalización no ha sido un proceso inclusivo ni bien equilibrado. Hay áreas en que se ha avanzado mucho y otras que exhiben grandes rezagos o retrocesos. Por ejemplo, la globalización de la volatilidad financiera ha afectado a muchos países de manera adversa, pero el grado de volatilidad depende de las reglas y regulaciones existentes en el mundo y también del manejo macroeconómico interno.

\section{El peso relativo de la globalización}

A continuación me refiero a tres áreas fundamentales de la globalización económica y a cómo ellas impactan a las economías nacionales.

El comercio ha crecido fuertemente desde fines de la segunda guerra mundial. La «torta» de lo que se produce 
en bienes y servicios a nivel mundial se va agrandando. Al mismo tiempo, dentro de cada país el componente que se dirige hacia el mercado externo se expande más rápidamente.

Entonces, podría pensarse que debido a que durante décadas las exportaciones han sido lo más dinámico, tenderían a abarcar casi toda la economía. Sin embargo, esto no ocurre porque el punto de partida de la producción exportada hacia mediados del siglo XX era muy reducido. Luego, aunque cre- ce muy rápidamente, sigue siendo un componente minoritario.

En efecto, de la producción total del mundo, la que cruza fronteras representa solamente 1 de cada 5 dólares, y 4 se quedan dentro de las fronteras nacionales (gráfico 2). ¿Cuál es la relación en los Estados Unidos? En la economía estadounidense, de cada 10 dólares que se producen, 1 sale en forma de exportaciones y los nueve restantes se transan entre sus 50 estados y no cruzan fronteras internacionales.

\section{GRÁFICO 2}

Mundo: PIB eXPortado y PIB NO EXPORTADO, 2004. (MILES DE MILLONES DE DÓLARES Y PORCENTAJE DEL PIB)

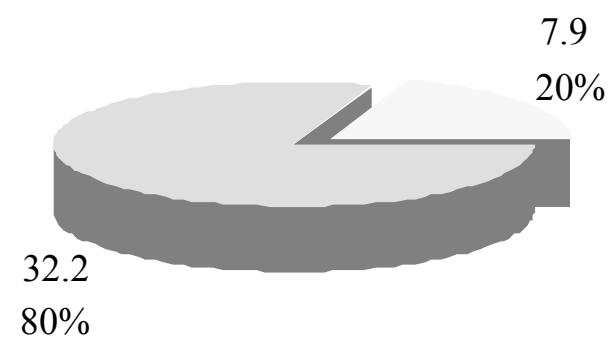

PIB no exportado PIB exportado

¿Qué indica esto? Sabemos que las exportaciones constituyen un aporte importante para el desarrollo, porque permiten aprovechar economías de escala y los recursos naturales. Se trata de un elemento muy positivo. Pero nunca debe actuarse como si las exportaciones fuesen lo único relevante. $\mathrm{Si}$ solamente nos preocupamos de apoyar a los exportadores y nos olvidamos del resto, estaremos dejando fuera del desarrollo a la mayor parte de la producción nacional.

Cuando examinamos la experiencia de países con éxito exportador encontramos, por ejemplo, el caso de Corea. En función de la cantidad de productos coreanos que llegan a todo el mun- 
do, podría pensarse que produce solo exportaciones. Es efectivo que ellas son significativas en su economía, pero solo representan algo más de $40 \%$ de su producto. Por lo tanto, en Corea todavía tiene más peso lo que no se exporta. Al analizar las claves de su desarrollo, de país pobre hacia 1965 a país cuyo ingreso por habitante duplica el de América Latina, encontramos que hubo dinamismo tanto en la producción de exportaciones como en el resto de la economía. Efectivamente, las exportaciones crecieron notablemente, $12 \%$ por año en promedio. ¿Qué pasó con el resto de su economía? La parte del PIB que no exporta, los bienes y servicios que producía y se usaban en los mercados nacionales, se expandió a un $6 \%$ anual durante un tercio de siglo. Se trata de una cifra positiva impresionante.

Para crecer, se necesita que el conjunto de los agentes de la economía esté trabajando a alta velocidad. Por lo tanto, debemos preocuparnos de crear condiciones amigables con todos los que producen, tanto para la exportación como para el mercado nacional, especialmente si los que producen para este último son la mayoría de los trabajadores, empresarios y firmas. Eso es lo que en términos técnicos se llama generar competitividad sistémica. Hay competitividad sistémica cuando el conjunto de la economía funciona crecientemente mejor y no existe solo un sector dinámico (que envía recursos naturales al exterior, por ejemplo), sino un avance armónico de los distintos sectores, con creciente valor agregado y capacitación de su gente, trabajadores y empresarios.

El segundo comentario se refiere a la inversión extranjera, en la región, que ha crecido vigorosamente durante los últimos dos decenios. En este período ella aumentó en promedio un $14 \%$ por año, mucho más que el producto y que el comercio mundiales.

El que la inversión extranjera sea tan vigorosa ¿significa que nuestro crecimiento depende esencialmente de esos flujos? Este depende solo parcialmente de la inversión extranjera directa (IED). Suele ser clave en las exportaciones y en los sectores de punta. Pero el gráfico 3 muestra que la IED representa solo un décimo de la inversión productiva mundial.

Podemos tomar el ejemplo de China, que en los últimos años ha sido el mayor receptor de inversión extranjera en el mundo. Podría pensarse que su gran crecimiento se basa mayoritariamente en la inversión extranjera. Pero no es así. Si observamos cuánto invierte en total China cada año y se compara con la inversión extranjera que recibe, se constata en que el último quinquenio solo el $12 \%$ de la inversión productiva se explica por la inversión extranjera. El $88 \%$ restante es inversión de los propios chinos. La inversión extranjera es muy relevante, pues ese $12 \%$ está muy presente en las exportaciones, pero China no depende solo de las exportaciones. Exporta mucho, pero el resto de la economía tiene mayor peso en su PIB. Por lo tanto, el bienestar de 
su gente y la recaudación tributaria de mente del desempeño del resto de su su gobierno dependen mayoritaria- economía.

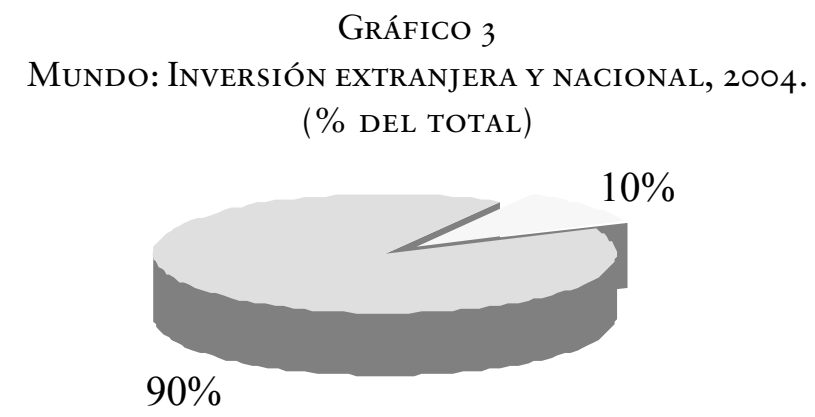

Inversión extranjera

Inversión nacional

Fuente: FMI

En cuanto a la inversión extranjera, debemos procurar atraerla, especialmente en las áreas más relevantes para la innovación, y que llegue a crear nueva capacidad productiva, en vez de concentrarse en la compra de lo ya existente.

En suma, es clave generar un buen entorno para la producción y la inversión productiva, y no mirar solo una fracción de la economía, pensando que lo importante es aquello que nos liga directamente a la economía globalizada. Lo relevante es cómo el país se integra en ese proceso. Para ello es crucial que el país se inserte en la economía mundial integrándose a si mismo, y no desintegrándose con un sector que corre rápido y dejando al grueso de la economía atrasada, excluida.

La IED, creadora de nueva capacidad, suele representar flujos estables. El equipo y la maquinaria, por ejemplo, es esencialmente inmóvil, tanto o más que el trabajo. Este último se puede cambiar de ciudad, pero los equipos y la maquinaria permanecen largo tiempo fijos en un lugar, como activos inmovilizados. Sin embargo, también existe otra clase de capital, el capital financiero, que es tremendamente móvil.

El mundo se ha movido, en los decenios recientes, hacia un fuerte dominio de los enfoques financieros. Esto me lleva al tercer aspecto que quiero comentar: el papel del capital financiero.

El capital financiero ha crecido a una velocidad notable. La magnitud de los flujos financieros opaca las demás transacciones. Los volúmenes transados diariamente en los mercados cambiarios del mundo son 40 veces superiores al valor de las exportaciones (o importaciones) de bienes y servicios tangibles. Es decir, la relación es de 40 a 1 . 
En términos simples, el flujo asociado al comercio de bienes y servicios sería, más o menos, de un dólar de comercio por dos dólares de mercado cambiario. Pero son 40 dólares. Esta relación tan desigual es reflejo de los fondos que se mueven varias veces al día, principalmente ajenos al comercio real. Esto resulta positivo desde el punto de vista de quien quiere operar en un mercado donde siempre es fácil comprar y vender; pero crea un problema para el resto de la economía, donde opera la abrumadora mayoría de las empresas y trabajadores. En los mercados financieros hay frecuentes «cambios de ánimo", que afectan las expectativas de los precios de, por ejemplo, el dólar, lo que provoca que los fondos que en un momento se dirigían a cierto mercado geográfico, se vayan abruptamente a otro. Entonces, sucede que una economía en desarrollo que está llena de dólares, al día siguiente puede encontrarse con gran escasez de ellos. Estos cambios de ánimo de los mercados financieros y cambiarios se sienten con mucha fuerza en la economía real, esto es, en la producción, el empleo, las utilidades y la recaudación fiscal.

Las crisis afectan la situación de las empresas, que bruscamente ven caer la demanda de sus productos. También afectan el empleo: por ejemplo, en América Latina la tasa promedio de desempleo aumentó en unos 3-4 puntos entre 1997 y 1999-2003. Así, las crisis dejan a los que producen riqueza -empresarios y trabajadores- en situaciones deprimidas, debilitadas. La globalización de la volatilidad financiera depende de las reglas y regulaciones existentes en el mundo y también del manejo macroeconómico interno.

Esto es así porque se trata de algo tangible, a diferencia de los flujos financieros que se aceleran o frenan en segundos: esto es lo que está detrás de las crisis financieras. Por ejemplo, Corea estaba lleno de capitales y, de repente, un día de 1997, se produjo un cambio de expectativas, y estos fondos emigraron bruscamente. Lamentablemente, el país ya había adecuado su economía a la abundancia de esos recursos y sufrió su abrupta escasez.

Desde el punto de vista de la equidad, cabe lamentar la creciente tendencia internacional a eximir de tributación a los capitales financieros o a tolerar la evasión tributaria. La consecuencia, gravemente regresiva, implica acentuar la carga tributaria sobre los que no pueden evitarla (o evadirla), los que no pueden huir. Esto es, el capital productivo y la mano de obra locales.

Hay una diferencia clara entre la inversión financiera y la inversión directa. Por eso aparece con fuerza en la literatura económica actual y en el enfoque de políticas en varios de los países que han actuado más responsablemente, estableciendo una diferenciación en el tratamiento de ambos flujos. Chile, por ejemplo, lo hizo al diseñar el encaje que en los noventa reguló la entrada de flujos financieros, de manera de promover la inversión extranjera directa y desalentar los flujos excesivos de carácter financiero. 


\section{Volatilidad financiera $y$ crisis regresivas}

Las crisis predominantes del mundo en desarrollo -el mundo emergente- desde 1982 hasta ahora, han estado asociadas principal aunque no exclusivamente a cambios de humor (apetito de riesgo en jerga financiera) de los mercados financieros y cambiarios. No es la inversión extranjera directa la que se va, ya que el grueso de su capital está inmovilizado en un yacimiento o en otra actividad productiva.

Un gran problema de la globalización financiera es su dimensión dinámica y volátil. Esto se aprecia cuando llegan grandes volúmenes de recursos líquidos a ciertas economías, provocando efectos desequilibradores. En muchas ocasiones estos flujos obedecen a desequilibrios macroeconómicos provocados por políticas inadecuadas. Las crisis de los últimos años -por ejemplo, las que ocurrieron en 1982, en 1985, en 1999, y en 2002 o 2003-, tuvieron origen financiero. La llegada de grandes flujos financieros inmediatamente antes de cada una de estas crisis abarata el dólar, infla la bolsa, y debilita el aparato productivo y la equidad en la sociedad. Luego, cuando se perciben riesgos, por ejemplo de devaluación o de caída de las bolsas, se registran salidas abruptas, con las correspondientes recesiones. Esto sucedió, nuevamente, en toda América Latina entre 1998 y 2003, cuando la región estuvo en recesión, y creció a una velocidad menor que la de la población.
La crisis incluso llegó a Chile, cuya economía estuvo en recesión (esto es, produciendo bajo su PIB potencial) desde 1999 hasta 2003. Esta inestabilidad macroeconómica constituye un problema de gran significación para el desarrollo democrático, para el crecimiento productivo y para la equidad.

Sin duda se requieren mayores volúmenes de capital que aquellos de que disponen nuestras economías. Pero lo que se necesita es capital que se destine a inversión productiva. En América Latina hemos vivido extensos períodos de inversión productiva deprimida, estrechamente asociados a situaciones recesivas. Con la recuperación reciente de la actividad económica, generalizada en casi toda la región, comienza a recuperarse la tasa de inversión a partir de los bajos niveles en que estaba situada. Es un hecho técnicamente demostrado, que no se puede dar bienestar y obtener desarrollo productivo sin equipos, maquinaria, infraestructura, construcciones comerciales y construcciones residenciales, y sin aumentar la productividad. Toda economía en desarrollo necesita expandir su capital físico para generar empleo productivo.

Cuando se deprime la formación de capital hay más incertidumbre y menos innovación, por lo tanto, menos tecnología. En este escenario, las que sufren las recesiones con mayor fuerza son las Pymes. El pequeño empresario se ve profundamente afectado, lo que se manifiesta en la escasa generación de empleo: en los años siguientes al contagio recesivo de 1999, la tasa de des- 
empleo en Chile se encumbró del $7 \%$ al $10 \%$. Esta crisis subsistió nada menos que seis años en el conjunto de la región. Lo prolongado de ella tiene que ver con la forma en que se interrelacionan los mercados externos y los mercados internos.

Para resolver los problemas planteados es de suma importancia alcanzar los objetivos planteados en la Declaración del Milenio, de las Naciones Unidas, que apuntan, entre otros aspectos, a que los países inmersos en la globalización puedan asumir iniciativas que promuevan el desarrollo productivo con equidad. Este es el medio de eliminar la pobreza 'estructural-mente'.

Para avanzar hacia soluciones desde la perspectiva internacional se han propuesto financiamientos innovado-res, que ayuden a los países a desarrollar políticas económicas y sociales equitativas, lo que en concreto significa darle un rostro social a la globali-zación. En la Cumbre de Monterrey, de las Naciones Unidas, se consensuó la necesidad de un financiamiento para el desarrollo mediante una serie de propuestas que permiten avanzar desde el financierismo y la volatilidad hacia el productivismo y mayor estabilidad real. Las Naciones Unidas están haciendo un monitoreo para lograr avances efectivos.

El mundo necesita más recursos para el desarrollo productivo y social. Requiere compromisos internacionales para que la gente pueda ganar con la globalización: ser beneficiario de ella y no solo un sujeto pasivo en la dimensión negativa. Se trata de contribuir a lograr que la gente sea mayoritariamente «ganadora» de bienestar económico y social.

Hoy existe más conciencia respecto de estos temas y se han generado múltiples acuerdos de todo tipo. Efectivamente, hay muchos acuerdos, pero la acción es insuficiente. Por otra parte, resulta evidente que la asistencia oficial para el desarrollo es inferior a lo comprometido por muchas de las naciones industrializadas. En consecuencia, es necesario pasar de las palabras a la acción. Y eso es lo que ha hecho un grupo de países. En 2004 dieron los primeros pasos, al identificar fuentes de recursos innovadoras, adicionales, que contribuyan a promover bienes públicos y el desarrollo económico, desalentando males públicos como el hambre y la pobreza. Los países participantes diseñaron un menú de opciones -no un modelo único- que permite dar cuenta de las diversas preferencias de los distintos países. En esa iniciativa me concentro a continuación.

\section{LA INICIATIVA DE ACCIÓN CONTRA EL HAMBRE Y LA POBREZA}

En los últimos años hemos conocido varias expresiones de colaboración internacional sustantiva en el marco de las Naciones Unidas. Allí se han adoptado compromisos para mejorar la gobernabilidad mundial y lograr un desarrollo que pueda beneficiar más equitativamente a todos. Los Objetivos 
de Desarrollo del Milenio y los acuerdos sobre Financiamiento del Desarrollo del Consenso de Monterrey son dos destacados ejemplos de este tipo de compromisos a nivel internacional.

Lo que ha faltado es una colaboración más vigorosa que se traduzca en acción. El mundo viene tomando cada vez más conciencia de esta forma de incumplimiento de los compromisos adquiridos, sobre todo respecto de los plazos establecidos para el logro de los primeros. Por otra parte, algo se ha avanzado en materia del segundo, pero el avance ha sido dispar y deja de lado áreas muy importantes.

En uno de los muchos y valiosos esfuerzos unilaterales y multilaterales emprendidos recientemente, en 2004 un grupo de países del norte y del sur decidieron unir fuerzas para contribuir a reducir la brecha existente entre grandes necesidades y escaso financiamiento. Para contribuir a "hacerlo realidad", pasando de las palabras a la acción.

Los presidentes de Brasil, Chile y Francia, junto al Secretario General de las Naciones Unidas, a los que después se sumaron los jefes de gobierno de España, Alemania, Argelia y Sudáfrica, lanzaron la Iniciativa para la Acción contra el Hambre y la Pobreza con el objetivo de identificar fuentes innovadoras de financiamiento. Este debía destinarse a iniciar proyectos piloto que facilitaran la consecución de los Objetivos de Desarrollo del Milenio. En este contexto, la Iniciativa recibió un fuerte respaldo de las Naciones Unidas y se constituyó un grupo técnico (GT-7) reducido, cuyos miembros son designados por los respectivos Jefes de Estado. Esta colaboración norte-sur identificó una serie de posibles fuentes innovadoras de financiamiento, como se puede apreciar en el Cuadro 1 (ver en la página siguiente).

Lo clave de esta asociación nortesur, con sustento técnico, con el impulso de los presidentes de los siete países, es que ha contribuido a esfuerzos dirigidos a corregir una globalización que se mueve hacia la vía deshumanizada y procíclica, así como a crear conciencia sobre esos temas e ir acompañando con hechos concretos las palabras de las cumbres internacionales. Ello se refleja en impulsos hacia una globalización que incluya, entre otras, medidas, gravámenes o tasas solidarias internacionales, y ayuden a crear espacios para que nuestros países puedan hacer mejores políticas nacionales. Esto es, reposicionar a la democracia, porque la democracia implica poder escoger el camino por el cual se avanza.

En 2006 se creó un grupo más amplio formado por estos siete países: el Grupo Piloto sobre Contribuciones de Solidaridad en Favor del Desarrollo, que integran actualmente 45 naciones del norte y del sur, de muy diversos niveles de desarrollo. Todos ellos han manifestado que están dispuestos a identificar y aplicar impuestos que puedan contribuir al desarrollo. Una contribución mediante la generación de financiamiento o desalentando «males públicos», como la evasión tributaria o las crisis financieras. 


\section{CuAdro I}

FUENTES DE FINANCIACIÓN INNOVADORAS PARA LA LUCHA CONTRA EL HAMBRE Y LA POBREZA: MENÚ DE OPCIONES DEL GRUPO TÉCNICO (GT-7)

- Tasas solidarias sobre pasajes aéreos

- Reducción de evasión tributaria y paraísos fiscales

- Incremento de los beneficios de las remesas (vínculo con micro-crédito)

- Tasas solidarias sobre transacciones financieras

- Tasas solidarias sobre comercio de armas

- Servicio financiero internacional (IFF)

- Emisión de Derechos Especiales de Giro contracíclicos

- Contribuciones voluntarias a través de tarjetas de crédito

- Inversión responsable desde el punto de vista social 0 «fondos éticos»

- Lotería solidaria

Ambos grupos comparten un enfoque amplio orientado a la búsqueda de la globalización de la solidaridad; de una globalización con rostro humano, que conduzca a un desarrollo capaz de beneficiar a todos nuestros países. En pro del desarrollo y en pro de la equidad de las fuentes de financiamiento y del uso de los recursos.

El GT-7 ha logrado avances reales. Efectivamente se han tomado medidas concretas para pasar de las palabras a la acción.

En Nueva York, en septiembre de 2004, se reunieron jefes de gobierno o ministros de la mayoría de los países del mundo, bajo la conducción de los presidentes Chirac, Lagos, Lula y Rodríguez Zapatero. Frente a las propuestas preparadas por el Grupo Técnico, en una Declaración sustantiva, 110 países expresaron su apoyo a que el grupo prosiguiese su labor orientada a la Cumbre Mundial sobre evaluación del grado de cumplimiento de los Objetivos de Desarrollo del Milenio, que debía realizarse en septiembre de 2005.

Así, con el impulso de Francia y el apoyo de Chile y Brasil, se avanzó en una propuesta de establecer una tasa sobre los pasajes aéreos internacionales. El 14 de septiembre de 2005 se presentó oficialmente el proyecto piloto sobre la contribución solidaria (impuesto/tasa) de esta naturaleza, cuyo producto sería destinado a la lucha contra el VIH/SIDA, la tuberculosis y el paludismo.

De hecho, en 2006, un grupo de países puso en marcha el proyecto piloto de acción contra las tres pandemias mencionadas. El proyecto, conocido como UNITAID, es un servicio internacional para la adquisición de medicamentos. Para evitar la duplicación de 
gastos administrativos funciona por intermedio de la Organización Mundial de la Salud. Esta característica del servicio, de evitar la burocracia y la duplicación de gastos, es un elemento que no puede ignorarse $\mathrm{y}$, de hecho, debe ser una de las principales condiciones del diseño y de la identificación de fuentes y de formas de utilización de los recursos.

UNITAID fue creado por Brasil, Chile, Francia, Noruega y el Reino Unido, y cuenta con el apoyo de las fundaciones Clinton y Gates.

Las tres pandemias ya mencionadas son importantes causas de hambre y pobreza en los hogares que son víctimas de ellas. Las tres son determinantes de los avances y retrocesos que se dan en el proceso de desarrollo. Fuera de la lucha frontal contra ellas, otro de los propósitos de UNITAID es perfeccionar el funcionamiento de los mercados, a fin de reducir el costo y elevar la calidad de los medicamentos, no solo en favor de sus beneficiarios directos sino también de los países en desarrollo en general.

La mayor parte del financiamiento del servicio proviene de un impuesto solidario aplicado a los vuelos aéreos internacionales. A la fecha, 25 países han informado que están considerando la posibilidad de aplicarlo o ya han comenzado a hacerlo, o bien de recurrir a otras fuentes de financiamiento sustentable. De hecho, a partir de octubre de 2006 están fluyendo contribuciones de un número creciente de países. Chile, desde marzo de 2006, y
Francia, desde julio de ese año, fueron los primeros en incluir en las tasas de embarque una suma destinada a esta iniciativa solidaria.

Alentamos a todos los Estados Miembros de las Naciones Unidas a considerar la posibilidad de contribuir a esta novedosa fuente de financiamiento, que tiene las siguientes características positivas:

i. el transporte aéreo de pasajeros es una de las actividades que se han visto favorecidas por la globalización;

ii. en general, el transporte aéreo es un sector que, proporcionalmente, paga pocos impuestos, por ejemplo, a los combustibles;

iii. es progresiva, en general, pues quienes hacen uso de servicios de transporte aéreo se encuentran entre los grupos de más altos ingresos y, por consiguiente, son buenos candidatos para que compartan parte de sus beneficios con el resto del mundo;

iv. en todos los casos en que se cobran impuestos en los aeropuertos su cobro es una operación desburocratizada y expedita.

¿Qué otras fuentes novedosas de financiamiento podrían considerarse? El grupo técnico ha estado trabajando en nueve propuestas, que van desde el impuesto solidario a los pasajes aéreos, ya mencionados, hasta la «securitización» de las remesas de los emigrantes y su vinculación a micro-créditos a los receptores. Debido a lo limitado del espacio, permítanme referirme solo a tres de ellas: 
Crisis externas y derechos especiales de giro (DEG)

El hambre y la pobreza están claramente relacionadas con las crisis monetarias derivadas del deterioro de los precios de exportación, de la salida y fuga de capitales, y de los desastres naturales. Estas crisis tienen graves efectos regresivos y son un fuerte inhibidor del desarrollo.

Con las recesiones, la gran mayoría pierde: pierden los trabajadores, los empresarios, el gobierno y, en suma, pierde el mundo. Es preciso contar con mecanismos eficaces de apoyo a los países en crisis, que muchas veces están haciendo su mejor esfuerzo pero sufren impactos externos, de términos del intercambio y en los precios de las exportaciones, que escapan a su control.

Hemos propuesto que se aprueben nuevas emisiones de derechos especiales de giro (DEG) por el Fondo Monetario Internacional (FMI). Los DEG son una moneda global especial que puede emitir el Fondo. ¿Dónde estamos hoy día al respecto? En 1997, durante la presidencia de Clinton, el Fondo Monetario aprobó una emisión de 22 mil millones de DEG, equivalentes a unos 34 mil millones de dólares. Falta que se haga efectiva. Después que los directores que representan a los países miembros en el FMI votaron en favor de esta emisión se inició el proceso de ratificación por parte de cada uno de los países. Hasta ahora lo han hecho 133 países, que tienen $77 \%$ del poder de votación total de la institución. Falta un voto, el del actual Gobierno de los Estados Unidos, que con $17 \%$ de los votos permitiría superar la mayoría de $85 \%$ requerida para que la emisión pueda realizarse.

La propuesta del GT-7 incluye la indicación de que los países desarrollados, que recibirían el $60 \%$ de los DEG que se emitan, los donen a un fondo contracíclico del mismo FMI o a mecanismos regionales o subregionales. $\mathrm{Su}$ propósito es que contribuyan a reducir los costos reales, económicos y sociales generados por las crisis financieras y la inestabilidad de los términos del intercambio.

Naturalmente, planteamos que los países afectados por estas crisis deben tener acceso a estos fondos, en condiciones revisadas ampliamentes, para que resulten efectivamente contracíclicos. Como ya se señaló, las crisis que han sufrido países en desarrollo por causas ajenas a su actuar constituyen un despilfarro económico.

\section{Evasión tributaria y paraísos fiscales}

El hambre y la pobreza también se relacionan con sistemas tributarios débiles debido, entre otros motivos, a la evasión tributaria a través de "paraísos fiscales».

Es un hecho grave que parte importante de la rentabilidad de los flujos financieros y de las ganancias de capi- 
tal involucradas en la globalización financiera no estén gravadas, o lo están insuficientemente. Una fuente de esta inequidad opera a través de paraísos fiscales.

La evasión tributaria involucra un aspecto ético, ya que castiga a los inversionistas legítimos, transparentes, abiertos, sin secretos, etc. que pagan sus impuestos, y beneficia a los que tienen malas prácticas, como el no pago de los impuestos, el lavado de dinero y el financiamiento del terrorismo, actividades que se financian con dinero derivado de prácticas ilegales o permitidas por vicios y errores de los sistemas tributarios. Las políticas permisivas en condiciones de auge de los flujos financieros han facilitado esta falla de la globalización. Lo que sabemos es que parte importante de recursos que se filtra de los sistemas tributarios de los países del norte y del sur se ampara en esos paraísos fiscales: es preciso ayudar a los países en desarrollo a fortalecer sus sistemas tributarios mediante la adopción de medidas que impidan la evasión a través de ellos. La evasión tributaria es muy injusta con los contribuyentes honrados. El Comité de Expertos sobre Cooperación Internacional en Cuestiones de Tributación de las Naciones Unidas, reformado recientemente, puede desempeñar un papel importante en este sentido y se debería estimular la interacción con las actividades de la OCDE, para ir avanzando hacia la adopción de medidas concretas contra la evasión tributaria internacional.
Debemos cooperar para movernos progresivamente, $\mathrm{y}$ «progresistamente», en ese ámbito. Es una de las prioridades fijadas por el GT-7 y por el grupo más amplio de 45 naciones.

Las entradas fiscales que produzca la lucha contra la evasión tributaria podrían asignarse -junto con otras posibles fuentes novedosas de financiamiento, tales como una lotería internacional- a un programa piloto destinado a reforzar la acción contra la desnutrición infantil. Brasil y Chile han estado estudiando el desarrollo de un programa piloto sobre la materia, como segunda área de financiación de la Iniciativa contra el Hambre y la Pobreza.

\section{Otros temas}

El grupo técnico y el Grupo Piloto sobre Contribuciones de Solidaridad a Favor del Desarrollo, encabezado por Noruega, sobre todo en la segunda reunión plenaria del Grupo Piloto (Oslo, febrero de 2007), han estado estudiando la posible adopción de un impuesto sobre las transacciones en divisas destinado al desarrollo, realizando al respecto un trabajo técnico consistente y responsable. Un impuesto reducido y que no tenga efectos distorsionantes podría aportar un volumen significativo de recursos para desarrollo. En vista de sus ventajas, estoy seguro de que esta posibilidad merece seria consideración. Un importante refuerzo técnico es un documento preparado por el destacado economista John Williamson 
para el GT-7². A este esfuerzo también han contribuido estudios de organizaciones no gubernamentales, tales como el Stamp Out Poverty, financiado por el gobierno de Noruega.

La evasión fiscal, las crisis monetarias y financieras y las pandemias tienen un alto costo social en términos de subdesarrollo, hambre y pobreza.

Muchos países y organizaciones han logrado adelantos en estos frentes, consistentes sobre todo en una mayor conciencia social y ética, mayor disposición política y mejor asistencia técnica para la adopción de medidas más eficaces. Ahora es posible avanzar mucho más rápido en la colaboración en pro de un desarrollo amplio y más equitativo. Podemos acelerar el avance en la dirección de la humanización, limitando la especulación y la desigualdad, al poner los recursos legítimos generados por la globalización a trabajar a favor de la estabilidad y del desarrollo con equidad.

2 Ver John Williamson, «Un impuesto sobre las transacciones cambiarias como instrumento de lucha contra la pobreza», Revista de la CEPAL, N 89, agosto de 2006. 